\title{
Research Progress on PATJ and Underlying Mechanisms Associated with Functional Outcomes After Stroke
}

\author{
Wen-jie Wang' \\ Tian-Jie Lyu ${ }^{2,3}$ \\ Zixiao $\mathrm{Li}^{1-5}$
}

'Vascular Neurology, Department of Neurology, Beijing Tiantan Hospital, Capital Medical University, Beijing, 100070, People's Republic of China: ${ }^{2}$ China National Clinical Research Center for Neurological Diseases, Beijing, 100070, People's Republic of China; ${ }^{3}$ National Center for Healthcare Quality Management in Neurological Diseases, Beijing, 100070, People's Republic of China; ${ }^{4}$ Chinese Institute for Brain Research, Beijing, 100070, People's Republic of China; ${ }^{5}$ Research Unit of Artificial Intelligence in Cerebrovascular Disease, Chinese Academy of Medical Sciences, Beijing, 100070, People's Republic of China
Correspondence: Zixiao Li

Department of Neurology, Beijing Tiantan Hospital, Capital Medical University, Chinese Institute for Brain Research, No. 119 West Road, Southern Fourth

Ring Road, Beijing, 100070, People's Republic of China

Tel +86-1059978350

Fax +86-1059973383

Email lizixiao2008@hotmail.com

\begin{abstract}
Cell polarity is an intrinsic property of epithelial cells regulated by scaffold proteins. The CRB (crumbs) complex is known to play a predominant role in the dynamic cooperative network of polarity scaffold proteins. PATJ (PALS1-associated tight junction) is the core component in the CRB complex and has been highly conserved throughout evolution. PATJ is crucial to several important events in organisms' survival, including embryonic development, cell polarity, and barrier establishment. A recent study shows that PATJ plays an important role in functional outcomes of stroke. In this article, we elaborate on the biological structure and physiological functions of PATJ and explore the underlying mechanisms of PATJ genetic polymorphism that are associated with poor functional outcomes in ischemic stroke.
\end{abstract}

Keywords: PATJ, cell polarity, stroke

\section{Introduction}

Cell polarity is known to be essential for cellular functions, ${ }^{1}$ especially in epithelial cells. $^{2}$ Research has shown that polarity protein complexes play an important role in the process of cell polarization ${ }^{3}$ and are involved in the formation of intercellular junction complexes. Cell polarity protein complexes mainly include the CRB (crumbs) - PALS1 (protein associated with LIN-7 1) -PATJ (PALS1-associated tight junction) complex, PAR3 (protease activated receptor 3) - PAR6 (protease activated receptor 6) - aPKC (atypical protein kinase C) complex at the top of the cell, and the SCRIB (scribble) - DLG (discs large) - LGL (lethal giant larvae) complex at the base (shown in Figure 1). These components cooperate with each other and participate in the establishment and stabilization of epithelial polarity. ${ }^{4}$ PATJ is an essential component of CRB complex. It is involved in a series of biological processes, such as cell migration, angiogenesis, and junction complex construction. It has also been found to be associated with tumorigenesis, ${ }^{5}$ viral infection $^{6}$ and other diseases.

Ischemic stroke is the most common fatal disease in China, and the majority of survivors will remain permanently disabled. Recently, increasing number of studies have focused on the clinical outcomes after stroke. The functional prognosis of stroke patients is not only affected by disease severity and rehabilitation strategy, but also partly attributed to inflammatory responses, genetics, blood-brain-barrier (BBB) permeability, collateral circulation and other factors. ${ }^{7}$ Combined with 


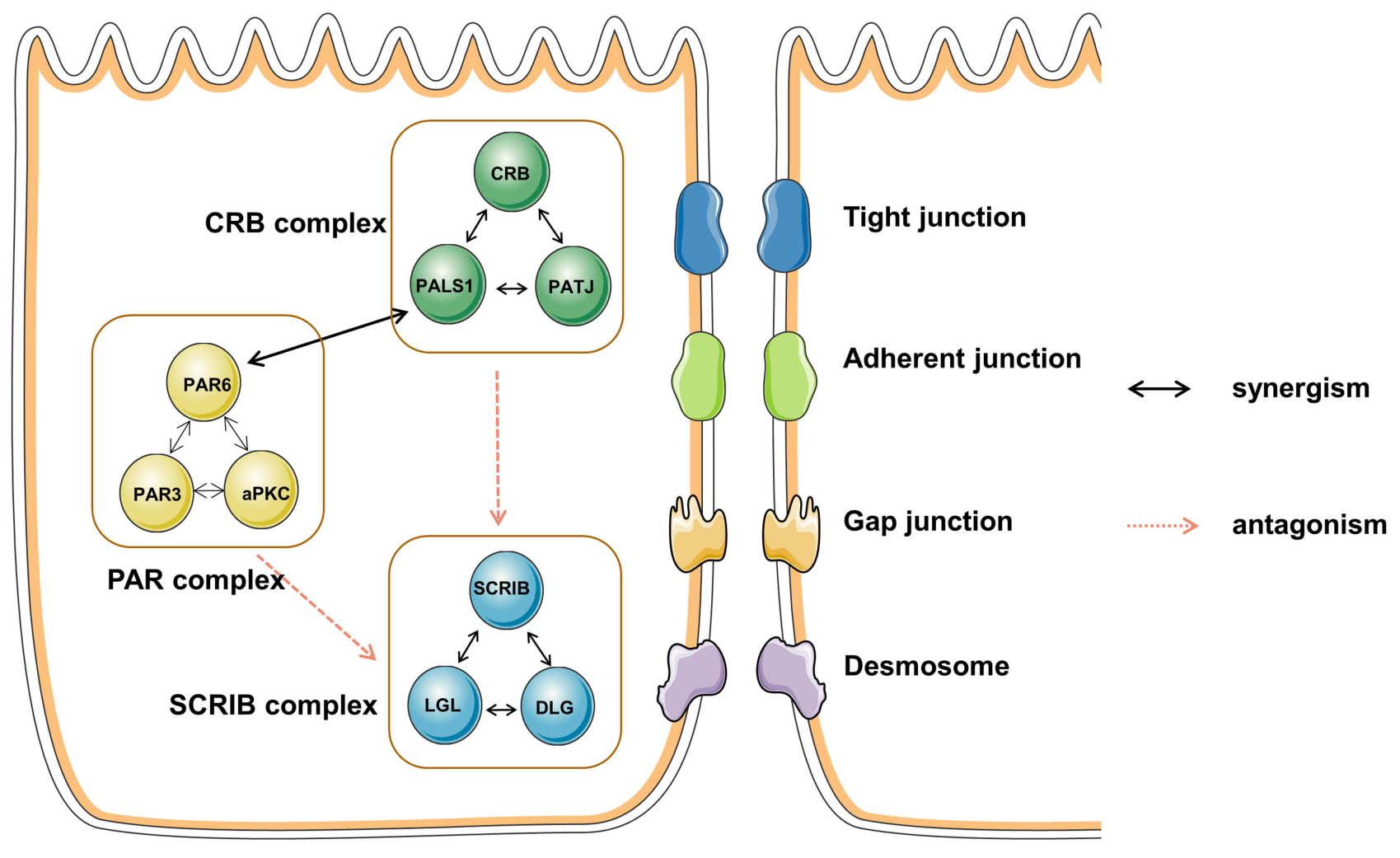

Figure I Dynamic cooperative network of polarity protein complex.

Abbreviations: CRB, crumb; PATJ, PALSI-associated tight junction; PALSI, protein associated with Lin-7 I; PAR6, protease activated receptor 6; PAR3, protease activated receptor 3; aPKC, atypical protein kinase C; SCRIB, scribble; LGL, lethal giant larvae; DLG, discs large.

physiological functions of PATJ, we hypothesized that PATJ may be an important predictor of stroke prognosis. This conjecture is supported by a largest genome-wide association study (GWAS) on ischemic stroke outcomes to date with 1225 stroke patients enrolled in 12 cohorts, in which Mola-Caminal and colleagues ${ }^{8}$ found that single nucleotide polymorphisms (SNPs) in PATJ were associated with poor prognosis at 3 months after a stroke. The top variant was rs76221407. This study was the first to demonstrate an association between PATJ and prognosis of ischemic stroke at a large-scale, suggesting that PATJ may be a new target for rehabilitation strategies after a stroke. However, the biological mechanism of PATJ in stroke is unknown. In this article, we briefly review the basic structure and physiological functions of PATJ and explore the potential mechanisms by which abnormal expression of PATJ may contribute to poor outcomes after stroke.

\section{PATJ and Its Homologue}

PATJ is a polar scaffold protein that interacts with other scaffold proteins (such as PALS1, CRB, etc.) through domains of protein-protein interaction to form homologous or heterologous protein complexes, participating in the establishment of cell polarity and performing corresponding biological functions. PATJ is widely distributed in the brain and skeletal muscle, and is located in different regions of cells during the process of cell polarization. PATJ localizes to the vacuolar apical compartment (VAC) in nonpolarized cells, and targets the apical region and tight junctions (TJs) during polarization to participate in the formation of intercellular junction structures.

The story of discovery of PATJ is interesting. PATJ was originally known as hINADL (human InaD like), and was initially considered to be a homologue of INADL (inactivation no after-potential D) in Drosophila. ${ }^{9}$ hINADL was thought to contribute to clustering membrane proteins. ${ }^{10}$ However, after the analysis of PATJ domains, it was found that the PDZ (postsynaptic density-95/diss large/ZO-1) domains of PATJ showed strong similarity to those domains of DLT (discs-lost) in Drosophila. The homology was more than $50 \%$. DLT, also known as DPATJ, ${ }^{11}$ is a cytoplasmic PDZ domain protein in Drosophila, which consists of an L27 (lin2 lin7 binding) domain at the N-terminal and four 


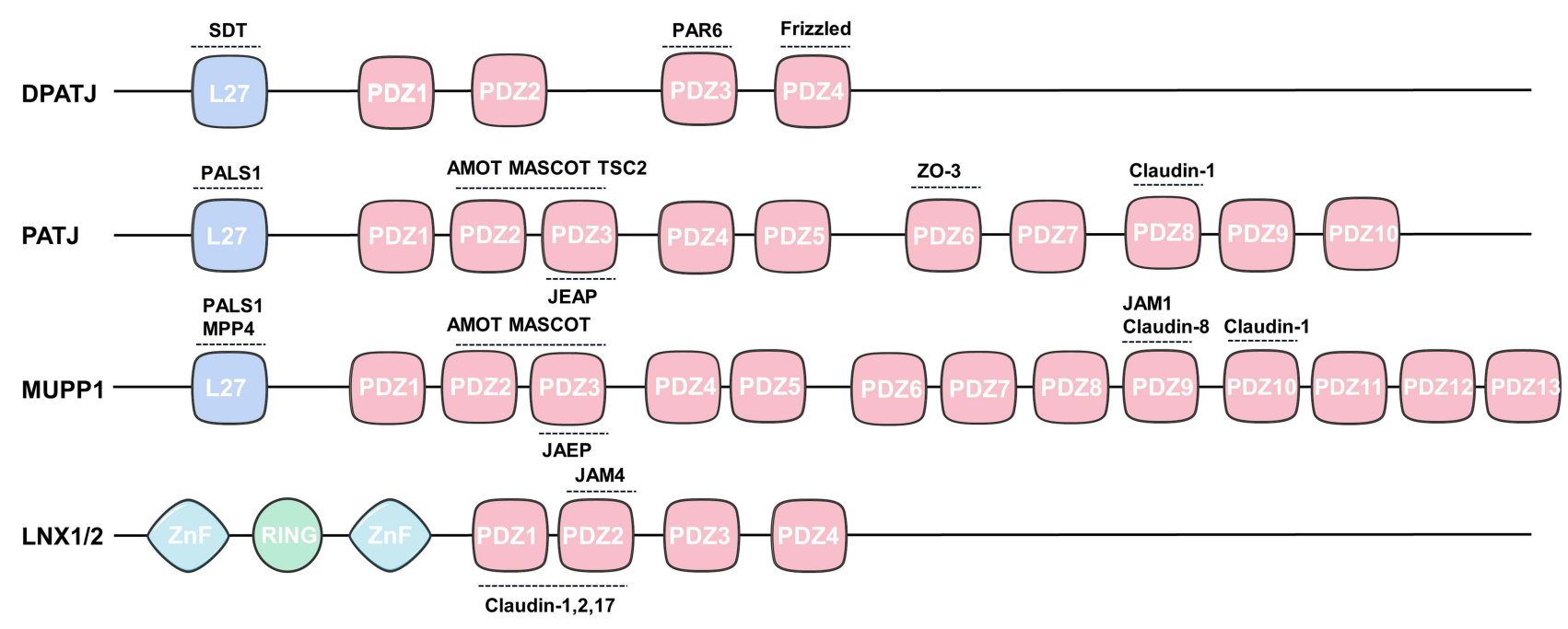

Figure 2 The structure of PATJ and its homologues.

Abbreviations: SDT, stardust; PAR6, protease activated receptor 6; PALSI, protein associated with LIN-7 I; PATJ, PALSI-associated tight junction; AMOT, angiomotin; MASCOT, TMAGI-I-associated coiled-coil tight junction protein; TSC2, the tuberous sclerosis 2 protein; JEAP, junction-enriched and -associated protein; JAMI, junctional adhesion molecule I; MUPPI, multiple PDZ domain protein I; LNXI/2, liver of NUMB protein XI and X2; L27, lin2 lin7 binding; PDZ, postsynaptic density-95/diss large/ ZO-I; ZnF, zinc finger motif; RING, really interesting new gene; JAM4, junctional adhesion molecule 4.

PDZ domains at the C-terminal. The two PDZ domains at the terminal are related to cell polarity ${ }^{12}$ (shown in Figure 2). Considering that the biological structures of DPATJ and hINADL look similar, and that they are both involved in the establishment and maintenance of cell polarity, scientists renamed hINADL to PATJ, and deemed that PATJ was the homologue of DPATJ in mammals.

PATJ contains one L27 domain and ten PDZ domains $^{13,14}$ (shown in Figure 2). Through the second and third PDZ domains, PATJ binds to the angiomotin (AMOT) - binding protein family to establish tight junctions and stabilize epithelial polarity. PATJ can also use the second PDZ domain to interact with the tuberous sclerosis 2 protein (TSC2), ${ }^{15}$ thus linking the mammalian target of rapamycin (mTOR) pathway with the CRB complex and supporting the hypothesis that cell polarity proteins could participate in signal transduction. The carboxyl termini of Zona occludens-3 (ZO-3) and claudin-1 interact with the sixth and eighth PDZ domain, respectively, suggesting that PATJ plays an important role in the establishment of TJs. The association between ZO-3 and PATJ may be crucial for the recruitment of PATJ and related proteins to TJs. PATJ without the sixth PDZ domain was found to be incorrectly located in the region far from TJs, while the loss of the claudin-1 binding domain did not affect PATJ localization. $^{12}$

Although we did not find relevant literatures about functions of PATJ in the pathological process of stroke, we noticed that MUPP1 (multi-PDZ domain protein), a homologue of PATJ in humans, has been proven to be related to central nervous system diseases such as congenital hydrocephalus ${ }^{16}$ and autism. ${ }^{17}$ MUPP1 contains one L27 domain and thirteen PDZ domains (as shown in Figure 2). PDZ domains in MUPP1 can also be combined with PALS1 and the AMOT family. But the affinity of MUPP1 for PALS1 and AMOTrelated proteins is much lower than that for PATJ. ${ }^{12,18}$ MUPP1 can participate in the construction of intercellular junction complexes by binding to the transmembrane protein JAM1 (junctional adhesion molecule 1) and claudin-8. ${ }^{19}$ Given the similar structures and biological functions of MUPP1 and PATJ, we speculate that PATJ may also play a role in the occurrence and development of nervous system diseases.

Other than DPATJ and MUPP1, other proteins with multiple PDZ domains as main functional units, such as LNX1 (liver of NUMB protein X1) and LNX2 (liver of NUMB protein X2), are also considered to be homolog of PATJ (as shown in Figure 2). They take part in the process of nerve signal transduction ${ }^{20}$ and tumorigenesis in the brain, ${ }^{21}$ further suggesting that PATJ may be involved in neurological disorders.

\section{Underlying Mechanisms of Association Between PATJ and Stroke Outcomes}

Ischemic stroke is a common disease in the world, characterized by high disability rate, high recurrence rate and high 
mortality. Clinical prognosis is a core issue in stroke research, with significant implications on survival and quality-of-life. Common risk factors of poor functional outcomes post-stroke include advanced age, smoking, hypertension and hyperlipidemia, the PATJ gene polymorphism is also correlated with clinical outcomes at 3 months post-stroke. However, the mechanisms are still unclear. Based on published experiments and clinical evidence, we preliminarily discuss the potential mechanisms through which PATJ could cause poor prognosis after a stroke (shown in Figure 3 ).

\section{PATJ Regulates Stroke Outcomes via Cell Polarization}

Cell polarization - the asymmetric distribution of cytoskeleton, proteins, and organelles to specific regions in cellsplays an important role in physiological and pathological processes such as angiogenesis, and homeostasis. ${ }^{22}$ Epithelial cells use cell polarity to form a barrier against bacteria and viruses to ensure homeostasis. Asymmetric division of nerve cells, cell migration, axon formation and signal transduction also depend on cell polarity. ${ }^{23}$ Abnormal cell polarity is an important pathological mechanism in central nervous system diseases. For example, abnormal expression of the polarity scaffold-protein LGL leads to neuronal cell migration disorder, resulting in epileptic seizures. ${ }^{24}$

It has been reported that the dpatj mutation in Drosophila could severely disrupt the apical localization of CRB, resulting in the loss of cell polarity. ${ }^{11,25,26}$ PATJ is the homologue of DPATJ in mammals, which has the similar structure and physiological functions to DPATJ. As such, it can also stabilize normal structure of polar protein complexes such as CRB and PAR complex, ${ }^{27,28}$ regulate the formation and maintenance of cell polarity, and participate in axon growth, angiogenesis, and cell migration. It follows that PATJ could potentially affect the functional prognosis of ischemic stroke through these pathways.

Axons are an essential part of signal transmission between neurons. Neuronal somas emit nerve impulses, which are transmitted through the axon to other neurons to carry out biological functions. Axons grow under the

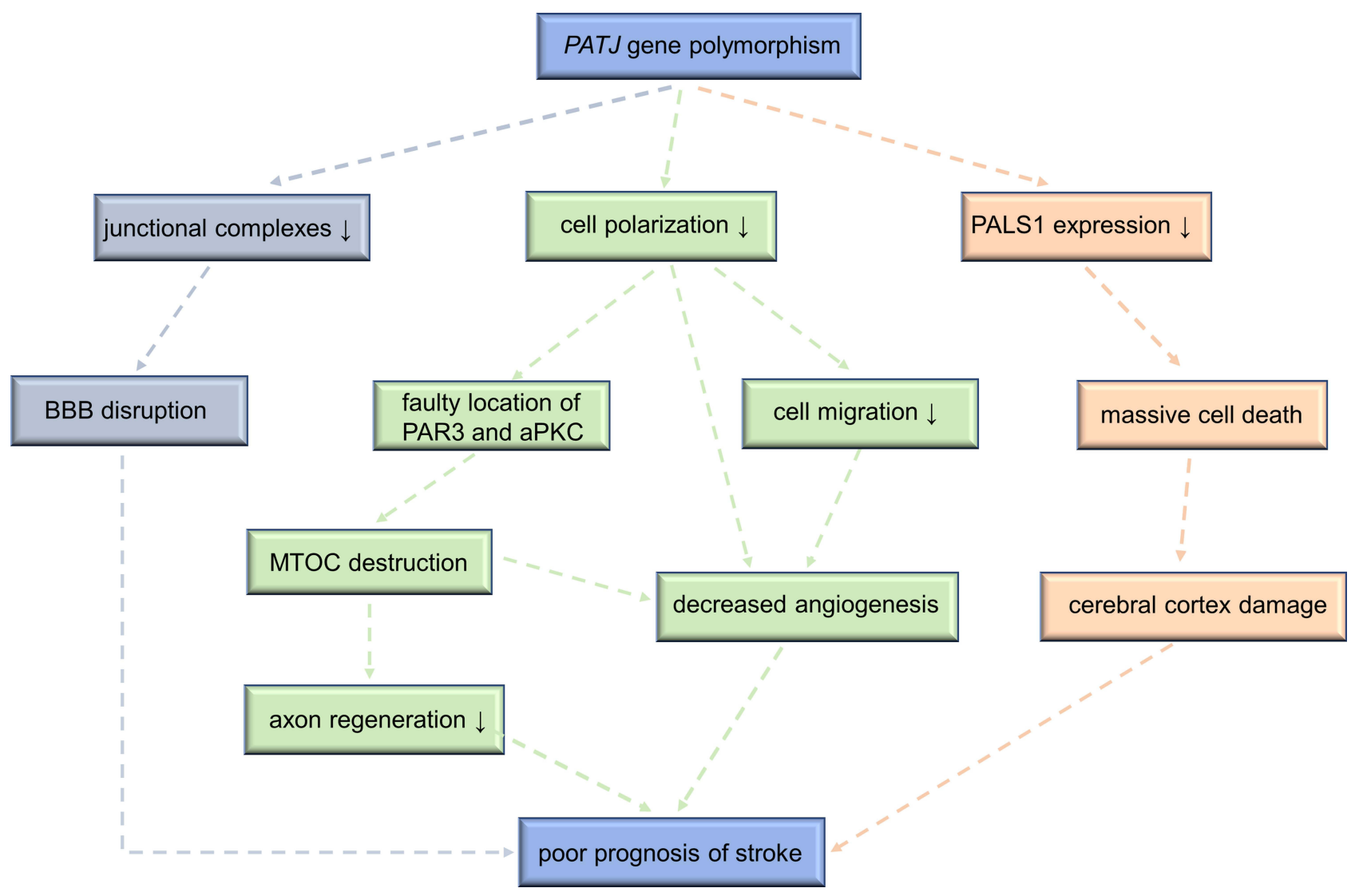

Figure 3 The underlying mechanisms that PATJ genetic mutations lead to poor prognosis of stroke.

Abbreviations: PATJ, PALSI-associated tight junction; PALSI, protein associated with Lin-7 I; PAR3, protease activated receptor 3; aPKC, atypical protein kinase C; BBB, blood-brain-barrier; MTOC, microtubule-organizing center. 
control of cell polarity and form synapses with dendrites after reaching the designated location, thus ensuring the accuracy of neuronal connections, material transport and signal transduction. ${ }^{29}$ Regeneration and repair of axons and dendrites are important for the functional recovery of the central nervous system. ${ }^{30}$ Microtubule-organizing center (MTOC) is involved in the formation of neuronal axons and dendrites, ${ }^{31}$ and the correct assembly of microtubules in the growth cone is the basis of neurite growth and extension. ${ }^{32}$ Abnormal expression of the PATJ gene leads to the faulty localization of polarity scaffold-protein PAR3 and $\mathrm{aPKC},{ }^{33}$ which affects the proper assembly of microtubule structure. This may explain why the PATJ gene polymorphism contributes to poor neurological recovery after a stroke. Although there are no basic experiments or clinical studies confirming that PATJ gene polymorphism is associated with axonal dysplasia, its homologues LNX1 and LNX2 have been shown to play an important role in synapse formation and signal transmission. ${ }^{20}$ Further studies are needed to confirm the relationship between PATJ and axons and dendrites.

Angiogenesis is a protective mechanism after a stroke. Neovascularization can mitigate hypoperfusion in ischemic brain tissue by increasing the supply of oxygen and nutrients, thus saving neurons in the ischemic penumbra. Cell polarity, which requires the participation of PATJ, is a prerequisite for angiogenesis. Under the control of cell polarity, single cells gather around the lumen to form the vascular wall, with the top of cells facing the lumen. However, in the PATJ RNA-knockout cell model, epithelial cells were distributed in a disordered manner and no angiogenesis was observed. After reintroducing PATJ back to the PATJ RNAi cell, the polarity defect was reversed and cells gathered to form the single-lumen polarized vessel. ${ }^{34}$ This suggests that PATJ plays a key role in angiogenesis by establishing and maintaining cell polarity. ${ }^{35}$

\section{Cell Migration is a Potential Mechanism Affecting Prognosis of Stroke}

Cell migration, also known as cell crawling, refers to cell movement caused by signals or changes in the concentration gradient of a substance. Cell migration is a common movement mode of cells, which is an important part of organismal growth and development. Angiogenesis is not only regulated by cell polarity, but also related to cell migration. In the process of angiogenesis, angioblasts migrate to specific locations, differentiate into endothelial cells, and then aggregate into cord-like structures composed of solid cell masses. After forming the vascular lumen, vascular cords form the primary vascular network.

PATJ mainly relies on MTOC to regulate cell migration. One of the characteristics of cell migration is the reorientation of MTOC to point in the correct direction of movement. In the PATJ knockout cell model, both PAR3 and aPKC in the PAR complex were not located at the leading edge of migrating cells, thus resulting in microtubule structure destruction and affecting the location of MTOC. ${ }^{33,36}$ Additionally, PATJ can regulate cell migration through binding to Ras homolog gene family member A (RhoA), ${ }^{37}$ spaghetti squash (SQH), ${ }^{38}$ kidney and brain expressed protein (KIBRA) ${ }^{39}$ and other molecules.

\section{Abnormal Expression of PATJ Affects the Formation of Junction Complexes}

Cell polarity is crucial for the normal function of most cells. It is characterized by the establishment of cell axes that allows different proteins and lipids to be asymmetrically distributed to specific regions, and subsequently performing their functions. There are junctional complexes such as TJs and adherens junctions (AJs) between polarized epithelial cells, which form physical barriers separating the top and base of the cell. TJs line the top of AJs, forming physical barriers that block soluble molecules through the intercellular spaces. The AJs can maintain normal tissue structures by mechanically connecting adjacent cells. Junctional complexes are considered to be indispensable for $\mathrm{BBB}$, and the permeability of $\mathrm{BBB}$ is related to functional prognosis of ischemic stroke. It is reported that BBB permeability is an independent predictor of ischemic stroke. Those with damaged BBB integrity have worse outcomes and poorer prognosis, independent of age, sex, infarct sizes and other factors. ${ }^{40}$

PATJ takes part in the establishment and maintenance of junctional complexes. Overexpression or deletion of PATJ in epithelial cells will destroy the specific localization of tight junction proteins, including $\mathrm{ZO}-1, \mathrm{ZO}-3$ and occludin, in the lateral membrane of cells; as well as affect the distribution of the CRB complex, leading to failure of PALS1 and CRB3 to gather at the tight junction. It has been suggested that PATJ is involved in the construction of tight junctions by linking polarity-proteins on the apical and basolateral membranes in cells. ${ }^{41-43}$ In a cell model with abnormal PATJ expression, the transepithelial 
resistance decreased significantly, reflecting the functional impairment of barrier formed by TJs. The downregulation of PALS1 was also observed. ${ }^{44}$ Normal functions of TJs were not recovered after supplementing PALS1, indicating that PATJ is irreplaceable in the formation and normal functioning of TJs.

PATJ also supports the stability of the AJs by regulating myosin activity. PATJ binds to the subunit of myosin phosphatase and reduces its ability to dephosphorylate, resulting in myosin activation. In PATJ-mutated cells, myosin is located in incorrect regions, and AJs disintegrate. ${ }^{38}$

Although there is no direct evidence that PATJ is related to the structures and functions of the BBB, there is evidence of such a relationship with MUPP1, which is thought to have similar physiological functions as PATJ. It is found that MUPP1 gene knockout mice had poor BBB integrity, ${ }^{16}$ suggesting that normal MUPP1 expression was the premise of maintaining BBB structures and functions. ${ }^{45}$ MUPP1 gene knockout also results in ependymocyte necrosis and astrocyte proliferation, leading to the stenosis of midbrain aqueduct and the obstruction of cerebrospinal fluid reflux. ${ }^{16}$ Hydrocephalus was induced in most mice and some died of cerebral hemorrhage. In this study, down-regulation of PALS1 levels and tight junction destruction were also observed.

Based on the theory that there are significant overlaps between the physiological functions of MUPP1 and PATJ, and that the relationship between PALS1, tight function and PATJ is closer than that with MUPP $1,{ }^{18}$ we infer that the PATJ gene polymorphism will damage the BBB due to cell junction destruction. This allows hyperactivated inflammatory substances to enter the brain, leading to an imbalance in central nervous system hemostasis and thus affecting post-stroke clinical outcomes. Abnormal PATJ expression can also cause hydrocephalus, cerebral hemorrhage and other adverse events, further contributing to the poor prognosis of stroke patients.

\section{PALSI Deficiency Links PATJ to Stroke Prognosis}

PALS1 is necessary for the development of the cerebral cortex. PALS1 is associated with the number of $\gamma$ aminobutyric acid (GABA) transporters, mTOR signal transmission, and primary cilia length in the central nervous system, ${ }^{46,47}$ thereby participating in the transduction of survival signals and the development of the nervous system. Conditional removal of PALS1 causes excessive generation of early-born postmitotic neurons followed by massive and rapid cell death, which destroys the structure of the cerebral cortex. ${ }^{4,46}$ Additionally, the levels of PATJ and PALS1 in cells are heavily influenced by one another. The integrity of the PATJ structure is key to the localization of PALS1 in the TJs. ${ }^{4,48}$ If the PATJ levels are low, PALS1 expression will be missing in the cell, while PATJ overexpression results in faulty localization of PALS1. Therefore, PATJ may indirectly affect nervous system development through regulating PALS1.

\section{Conclusion}

PATJ is an important cellular polar scaffold protein and is crucial for cell polarization, cell migration, and bloodbrain-barrier formation among other functions. PATJ gene polymorphism may lead to poor functional outcomes in stroke patients through inhibiting axon regeneration and angiogenesis as well as destroying blood-brain-barrier integrity. Basic science experiments and clinical studies are needed to explore possible reasons why the PATJ gene polymorphism contributes to poor prognosis of stroke and determine whether it could be a potential target in stroke diagnosis and treatment.

\section{Acknowledgments}

This work was jointly supported by Ministry of Science and Technology of the People's Republic of China (National Key R\&D Programme of China, 2017YF C1310901) and Natural Science Foundation of Beijing Municipality (Z200016).

\section{Disclosure}

The authors report no conflicts of interest in this work.

\section{References}

1. Zhang J, Yang X, Wang Z, et al. Structure of an L27 domain heterotrimer from cell polarity complex Patj/Pals1/Mals2 reveals mutually independent L27 domain assembly mode. J Biol Chem. 2012;287 (14):11132-11140

2. Shahab J, Tiwari MD, Honemann-Capito M, Krahn MP, Wodarz A. Bazooka/PAR3 is dispensable for polarity in Drosophila follicular epithelial cells. Biol Open. 2015;4(4):528-541. doi:10.1242/bio.20 1410934

3. Tan B, Yatim SM, Peng S, Gunaratne J, Hunziker W, Ludwig A. The mammalian crumbs complex defines a distinct polarity domain apical of epithelial tight junctions. Curr Biol. 2020;30(14):2791-2804.e6. doi:10.1016/j.cub.2020.05.032

4. Hakanen J, Ruiz-Reig N, Tissir F. Linking cell polarity to cortical development and malformations. Front Cell Neurosci. 2019;13:244.

5. Duning K, Rosenbusch D, Schlüter M, et al. Polycystin-2 activity is controlled by transcriptional coactivator with PDZ binding motif and PALS1-associated tight junction protein. J Biol Chem. 2010;285 (44):33584-33588. 
6. Assemat E, Crost E, Ponserre M, Wijnholds J, Le Bivic A, MasseyHarroche D. The multi-PDZ domain protein-1 (MUPP-1) expression regulates cellular levels of the PALS-1/PATJ polarity complex. Exp Cell Res. 2013;319(17):2514-2525. doi:10.1016/j.yexcr.2013.07.011

7. Kessler T, Schunkert H. Genetics of recovery after stroke a first step is done. Circ Res. 2019;124(1):18-20. doi:10.1161/CIRCRESAHA. 118.314269

8. Mola-Caminal M, Carrera C, Soriano-Tárraga C, et al. PATJ low frequency variants are associated with worse ischemic stroke functional outcome. Circ Res. 2019;124(1):114-120. doi:10.1161/ CIRCRESAHA.118.313533

9. Lemmers C, Médina E, Delgrossi MH, Michel D, Arsanto JP, Le Bivic A. hINADl/PATJ, a homolog of discs lost, interacts with crumbs and localizes to tight junctions in human epithelial cells. J Biol Chem. 2002;277(28):25408-25415. doi:10.1074/jbc.M202 196200

10. Philipp S, Flockerzi V. Molecular characterization of a novel human PDZ domain protein with homology to INAD from Drosophila melanogaster. FEBS Lett. 1997;413(2):243-248. doi:10.1016/S00145793(97)00877-6

11. Bhat MA, Izaddoost S, Lu Y, Cho KO, Choi KW, Bellen HJ. Discs lost, a novel multi-PDZ domain protein, establishes and maintains epithelial polarity. Cell. 1999;96(6):833-845. doi:10.1016/S00928674(00)80593-0

12. Bazellieres E, Assemat E, Arsanto JP, Le Bivic A, Massey-Harroche D. Crumbs proteins in epithelial morphogenesis. Front Biosci. 2009;14:2149-2169. doi:10.2741/3368

13. Wells C, Fawcett J, Traweger A, et al. A Rich1/Amot complex regulates the Cdc42 GTPase and apical-polarity proteins in epithelial cells. Cell. 2006;125(3):535-548.

14. Roh M, Makarova O, Liu C, et al. The maguk protein, Pals1, functions as an adapter, linking mammalian homologues of crumbs and discs lost. J Cell Biol. 2002;157(1):161-172.

15. Wen J, Wang Y, Chan C, et al. DrosophilaAtg9 antagonizes TOR signaling to regulate intestinal cell growth and epithelial homeostasis in Drosophila. Elife. 2017;6:e29338.

16. Feldner A, Adam MG, Tetzlaff F, et al. Loss of Mpdz impairs ependymal cell integrity leading to perinatal-onset hydrocephalus in mice. EMBO Mol Med. 2017;9(7):890-905. doi:10.15252/emmm.20 1606430

17. Tanabe Y, Fujita-Jimbo E, Momoi MY, Momoi T. CASPR2 forms a complex with GPR37 via MUPP1 but not with GPR37(R558Q), an autism spectrum disorder-related mutation. $J$ Neurochem. 2015;134 (4):783-793. doi:10.1111/jnc. 13168

18. Sugihara-Mizuno Y, Adachi M, Kobayashi Y, et al. Molecular characterization of angiomotin/JEAP family proteins: interaction with MUPP1/Patj and their endogenous properties. Genes Cells. 2007;12 (4):473-486

19. Roh M, Liu C, Laurinec S, Margolis BJ. The carboxyl terminus of zona occludens-3 binds and recruits a mammalian homologue of discs lost to tight junctions. $J$ Biol Chem. 2002;277(30):27 501-27509.

20. Young PW. LNX1/LNX2 proteins: functions in neuronal signalling and beyond. Neuronal Signal. 2018;2(2):Ns20170191. doi:10.1042/ NS20170191

21. Blom T, Roselli A, Tanner M, Nupponen NN. Mutation and copy number analysis of LNX1 and Numbl in nervous system tumors Cancer Genet Cytogenet. 2008;186(2):103-109. doi:10.1016/j. cancergencyto.2008.07.003

22. Banda E, McKinsey A, Germain N, Carter J, Anderson NC, Grabel L. Cell polarity and neurogenesis in embryonic stem cell-derived neural rosettes. Stem Cells Dev. 2015;24(8):1022-1033. doi:10.1089/scd.2014.0415

23. Piroli ME, Blanchette JO, Jabbarzadeh E. Polarity as a physiological modulator of cell function. Front Biosci. 2019;24(3):451-462. doi: $10.2741 / 4728$
24. Liu F, Du C, Tian X, et al. A novel LGI1 missense mutation causes dysfunction in cortical neuronal migration and seizures. Brain Res. 2019;1721:146332. doi:10.1016/j.brainres.2019.146332

25. Zhou WK, Hong Y. Drosophila Patj plays a supporting role in apical-basal polarity but is essential for viability. Development. 2012;139(16):2891-2896. doi:10.1242/dev.083162

26. Nam S, Choi KJ. Domain-specific early and late function of Dpatj in Drosophila photoreceptor cells. Dev Dyn. 2006;235(6):1501-1507.

27. Sen A, Sun R, Krahn MP. Localization and function of Pals1-associated tight junction protein in drosophila is regulated by two distinct apical complexes. J Biol Chem. 2015;290 (21):13224-13233. doi:10.1074/jbc.M114.629014

28. Koch L, Feicht S, Sun R, Sen A, Krahn MJ. Domain-specific functions of Stardust in embryonic development. Royal Soc Open Sci. 2016;3(11):160776.

29. Ribeiro LF, Verpoort B, de Wit J. Trafficking mechanisms of synaptogenic cell adhesion molecules. Mol Cell Neurosci. 2018;91:34-47. doi:10.1016/j.mcn.2018.04.003

30. Hansen AH, Duellberg C, Mieck C, Loose M, Hippenmeyer S. Cell polarity in cerebral cortex development-cellular architecture shaped by biochemical networks. Front Cell Neurosci. 2017;11:176. doi:10.3389/fncel.2017.00176

31. Jossin Y. Molecular mechanisms of cell polarity in a range of model systems and in migrating neurons. Mol Cell Neurosci. 2020;106:103503.

32. van de Willige D, Hoogenraad CC, Akhmanova A. Microtubule plus-end tracking proteins in neuronal development. Cell Mol Life Sci. 2016;73(10):2053-2077.

33. Shin K, Wang Q, Margolis B. PATJ regulates directional migration of mammalian epithelial cells. EMBO Rep. 2007;8(2):158-164. doi:10.1038/sj.embor.7400890

34. Shin K, Straight S, Margolis B. PATJ regulates tight junction formation and polarity in mammalian epithelial cells. J Cell Biol. 2005;168 (5):705-711. doi:10.1083/jcb.200408064

35. Jiang X, An W, Yang X, et al. Asymmetric distribution of CRUMBS polarity complex proteins from compacted 8-cell to blastocyst stage during mouse preimplantation development. Gene Expr Patterns. 2018;27:93-98.

36. $\mathrm{Du} \mathrm{D}, \mathrm{Xu} \mathrm{F}, \mathrm{Yu} \mathrm{L}$, et al. The tight junction protein, occludin, regulates the directional migration of epithelial cells. Dev Cell. 2010;18(1):52-63.

37. Lennon F, Mirzapoiazova T, Mambetsariev N, Mambetsariev B, Salgia R, Singleton PJ. Transactivation of the receptor-tyrosine kinase ephrin receptor A2 is required for the low molecular weight hyaluronan-mediated angiogenesis that is implicated in tumor progression. J Biol Chem. 2014;289(35):24043-24058.

38. Sen A, Nagy-Zsvér-Vadas Z, Krahn MP. Drosophila PATJ supports adherens junction stability by modulating myosin light chain activity. J Cell Biol. 2012;199(4):685-698. doi:10.1083/jcb.201206064

39. Duning K, Schurek E, Schlüter M, et al. KIBRA modulates directional migration of podocytes. J Am Soc Nephrol. 2008;19(10):1891-1903.

40. Nadareishvili Z, Simpkins AN, Hitomi E, Reyes D, Leigh R. Poststroke blood-brain barrier disruption and poor functional outcome in patients receiving thrombolytic therapy. Cerebrovasc Dis. 2019;47(34):135-142. doi:10.1159/000499666

41. Michel D, Arsanto JP, Massey-Harroche D, Beclin C, Wijnholds J, Le Bivic A. PATJ connects and stabilizes apical and lateral components of tight junctions in human intestinal cells. $J$ Cell Sci. 2005;118 (17):4049-4057. doi:10.1242/jcs.02528

42. Poliak S, Matlis S, Ullmer C, Scherer SS, Peles E. Distinct claudins and associated PDZ proteins form different autotypic tight junctions in myelinating Schwann cells. J Cell Biol. 2002;159(2):361-372. doi: $10.1083 /$ jcb. 200207050

43. Assemat E, Bazellieres E, Pallesi-Pocachard E, Le Bivic A, MasseyHarroche D. Polarity complex proteins. Biochim Biophys Acta Biomembr. 2008;1778(3):614-630. doi:10.1016/j.bbamem.2007.08.029 
44. Straight SW, Shin K, Fogg VC, et al. Loss of PALS1 expression leads to tight junction and polarity defects. Mol Biol Cell. 2004;15 (4):1981-1990. doi:10.1091/mbc.e03-08-0620

45. Yang J, Simonneau C, Kilker R, et al. Murine MPDZ-linked hydrocephalus is caused by hyperpermeability of the choroid plexus. EMBO Mol Med. 2019;11(1):e9540. doi:10.15252/emmm.201809540.

46. Kim S, Lehtinen MK, Sessa A, et al. The apical complex couples cell fate and cell survival to cerebral cortical development. Neuron. 2010;66(1):69-84. doi:10.1016/j.neuron.2010.03.019
47. McHugh EM, Zhu W, Milgram S, Mager S. The GABA transporter GAT1 and the MAGUK protein Pals1: interaction, uptake modulation, and coexpression in the brain. Mol Cell Neurosci. 2004;26(3):406-417. doi:10.1016/j.mcn.2004.03. 006

48. Nam SC, Choi KW. Interaction of Par-6 and crumbs complexes is essential for photoreceptor morphogenesis in drosophila. Development. 2003;130(18):4363-4372. doi:10.1242/dev.00648

\section{Publish your work in this journal}

Neuropsychiatric Disease and Treatment is an international, peerreviewed journal of clinical therapeutics and pharmacology focusing on concise rapid reporting of clinical or pre-clinical studies on a range of neuropsychiatric and neurological disorders. This journal is indexed on PubMed Central, the 'PsycINFO' database and CAS, and is the official journal of The International Neuropsychiatric Association (INA). The manuscript management system is completely online and includes a very quick and fair peer-review system, which is all easy to use. Visit http://www.dovepress.com/testimonials.php to read real quotes from published authors. 